\title{
Martensite Transformation Intergrain and Intragrain Autocatalysis in Fe-Ni alloys
}

\author{
José Roberto Costa Guimarães ${ }^{a}$, Paulo Rangel Rios ${ }^{a}$ * \\ ${ }^{a}$ Escola de Engenharia Industrial Metalúrgica de Volta Redonda - EEIMVR, Universidade Federal \\ Fluminense - UFF, Av. dos Trabalhadores, 420, 27255-125, Volta Redonda, RJ, Brasil.
}

Received: November 26, 2016; Revised: July 05, 2017; Accepted: July 28, 2017

\begin{abstract}
This work presents a consolidated view of the autocatalysis, thermal and entropic effects in martensitic transformation of polycrystalline $\mathrm{Fe}-31 \mathrm{wt} \% \mathrm{Ni}-0.02 \mathrm{wt} \% \mathrm{C}$, which has been a standard material for the investigation of fundamental aspects of the martensite transformation. The present work is based on the description of classical microstructure descriptors of the transformation and on generally accepted concepts regarding the martensitic transformation in iron base alloys. Present work agrees with the view that the autocatalysis is a means by which the martensite transformation promotes further nucleation and growth. Autocatalysis induces the nucleation and growth of secondary plates in addition to the relatively small number of primary nucleation sites and their corresponding primary plates. We demonstrate that autocatalysis can be factored out into intragrain and intergrain components. The analysis of these factors establishes that intragrain autocatalysis is athermal but intergrain autocatalysis possess an Arrhenius temperature dependence. The reasons for such a behavior are discussed in detail.
\end{abstract}

Keywords: metals and alloys, microstructure, martensite transformation, iron-nickel alloys, autocatalysis.

\section{Introduction}

Modern steels derive special characteristics from the martensitic transformation induced at different stages of processing and/or utilization. Properties improvement are generally obtained by designing the steel chemistry and processing to achieve the desired thermodynamic, crystallographic and kinetics aspects of the transformation ${ }^{1}$. In most steels, martensite transformation is admittedly nonthermally-activated ("athermal"), advancing as the driving force (negative of Gibb's Free Energy) increases. However, under certain conditions, time-dependent, thermally-activated martensitic transformation entails practical applications ${ }^{2}$.

Martensite transformation is diffusionless/displacive. Atomic displacements are less than an inter-atomic distance. The transformation is heterogeneous and initiates at scarce austenite sites $^{3}$ where thermal atomic mobility is somehow inhibited as discussed in ${ }^{4}$. The development of multi-variant nuclei therein yields the transformation ${ }^{5,6}$. Martensite propagates rapidly ${ }^{7,8}$, however, its displacive character prevents martensite crossing high-angle boundaries. Its growth is frequently halted by impingement. Impingements between martensite plates generally yield autocatalytic nucleation events that speeds up the transformation? ${ }^{7}$. These specificities are the sources of unique microstructure/property relationships that continue to be used to develop engineering materials.
In the present work, we revisit the martensite transformation in $\mathrm{Fe}-31 \mathrm{wt} \% \mathrm{Ni}-0.02 \mathrm{wt} \%$ alloy ${ }^{9}$ to expand the analysis of its autocatalytic aspects. Admittedly the transformation is non-thermal activated ("athermal") and develops at sub-zero temperatures that permits microstructural characterizations by up-quenching to room temperature. Our reference alloy, Fe-31wt $\% \mathrm{Ni}-0.02 \mathrm{wt} \% \mathrm{C}$, has been a standard material to research basic aspects of the martensitic transformation in iron base alloys. The present work analyzes in-depth the autocatalytic aspect of the transformation inferred from the observed microstructure.

\section{Background}

It is well-known that the initiation of the martensitic transformation in high Ni Fe-Ni-C alloys demands significant super-cooling to reach the martensite start temperature, $M_{S}$, frequently signaled by a transformation-burst, heat-rise and sonic-emission ${ }^{7}$. Phenomenologically, the martensite autocatalysis has been factored into intergrain and intragrain components, early observed and named "spread" and "fill-in"10. Fig.1 shows a microstructure typical of the initial transformation burst in polycrystalline $\mathrm{Fe}-31 \mathrm{wt} \% \mathrm{Ni}-0.02 \mathrm{wt} \% \mathrm{C}$. In polycrystalline materials, the intragrain transformation is delimited by internal boundaries not compatible with martensite's displacive character. Martensite impingement 
on grain boundaries can induce transformation within an adjacent grain ${ }^{10}$, yielding clustered grains as seen in ${ }^{9}$ Figs. 1a. Fig. 1b shows an example of a plate inducing the formation of another plate on a neighbor grain. One may consider each cluster as an individual "spread-event", which results from intergrain autocatalysis initiated by intragrain transformation in one grain. The intragrain transformation develops into zigzagging units that result from the optimization of transformation strains by the mutual accommodation of the martensite shape change as discussed in $^{11-13}$.

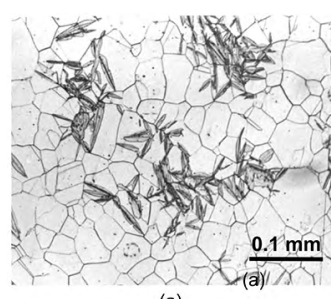

(a)

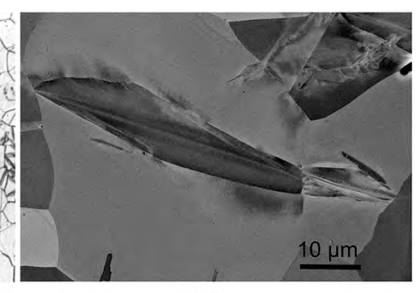

(b)
Figure 1. Microstructure of $\mathrm{Fe}-31 \mathrm{wt} \% \mathrm{Ni}-0.02 \mathrm{wt} \% \mathrm{C}$ transformed at the martensite start temperature: (a) Optical micrograph showing the isolated spread events ${ }^{9}$; (b) Scanning electron micrograph using backscattered electrons showing one martensite plate hitting the grain boundary and inducing the formation of another plate located within a neighbor grain; micrograph is a courtesy of Kahl Zilnyk.

\section{Experimental Details}

The material preparation is duly described in the referenced papers, so that they were not detailed here for brevity sake. The compiled data is normally obtained by systematic intercept and point counting on polished sections ${ }^{14}$. Fullman's method ${ }^{15}$ was used to estimate the number density of the martensite units. Since the original data did not display error bars, our experience with the quantitative stereological techniques used in the referenced works puts the relative errors from 5 to $20 \%$. The higher errors apply to the determination of the number density of the martensite units. These ascribed errors are significantly larger than the small variations incurred in the scanning/digitizing process used to collect the original data in the referenced papers.

The compiled database is typical of the Fe-31wt\%Ni$0.02 \mathrm{wt} \%$ alloy transformed by direct-quenching into baths (volume of the bath typically 1000 larger than the specimen size) of ethanol pre-cooled to sub-zero temperatures. The transformation temperatures were the temperatures of the baths. The following parameters were used to describe the microstructure of the martensite spread in Fe-31wt\%Ni$0.02 \mathrm{wt}^{\%} \mathrm{C} \mathrm{C}^{9}$. The volume fraction of martensite transformed, $V_{V M}$, and the number of martensite units transformed per unit volume of material, $N_{V M}$. The spread-events, tantamount the "spread" of the transformation were characterized by the volume fraction of material in transformed grains, $V_{V G}$, and the area per unit volume of the austenite grain boundaries between the transformed and untransformed grains, $S_{V G}$, regarding the "spread-events".

\section{Analytical Model}

The fast transformation-rate is a handicap to study the evolution of a martensitic transformation. To cope with this situation, we worked-out a formal model to delve into the martensite autocatalysis based on descriptors of the observed microstructure. The model's fitting-parameters are used to discuss the autocatalytic process. The validation of the formalism is based on the data fitting correlation. In addition, the specimens were transformed by quenching instead of by continuous (slow) cooling below $M_{S}$.

The model's concepts were introduced before ${ }^{16,17}$. Here we review and re-derive the equations. For this matter, consider that the martensite transformation initiates heterogeneously at $n_{V}$ randomly dispersed nucleation sites located within the material (austenite). As already mentioned the spread-event is promoted by intergrain autocatalysis and results in clusters of transformed grains as observed in present Fig. 1. Noteworthy, the development of a spread event does not involve the motion of the austenite grain boundaries. In absence of impingement between spread-events, it admissible that equal-sized spread-events, e.g. containing $\gamma$ grains, should be observed. Thus, ignoring superposition, the extended volume fraction, $V_{V G X}$, of material in spread-events is

$$
V_{V G X}=Q \cdot n_{V}
$$

where $Q=q \cdot \gamma$ is the total volume of a spread-event in absence of impingement and $q$ is the mean grain volume. Supposing that the spread-events inherit the uniform randomness of the $n_{V}$ nucleation $\operatorname{sites}^{3}$, one may use the well-known $\mathrm{JMAK}^{18-21}$ results to relate the extended and real volume fraction, $V_{V G}$, of the spread-events

$$
V_{V G}=1-\exp \left(-Q \cdot n_{V}\right)
$$

The extended interfacial area density of $n_{V}$ spread events ${ }^{17}$ is

$$
S_{V G X}=\phi(Q)^{2 / 3} \cdot n_{V}
$$

where $\varphi$ is a shape factor that is equal to $16 / 3$ in the case of tetrakaidecahedron. Substituting Eq. (1) into Eq. (3) gives

$$
\frac{V_{V G X}}{S_{V G X}}=\phi^{-1} \cdot Q^{1 / 3}
$$

In order to use microstructure descriptors to calculate $Q$ one may replace $V_{V G X}$ by $\ln \left(1-V_{V G}\right)^{-1}$ and $S_{V G X}$ by $S_{V G}$. $\left(1-V_{V G}\right)^{-1}$ in $^{22}$ Eq. (5)

$$
Q=\left(\phi \cdot\left(1-V_{V G}\right) \ln \left(1-V_{V G}\right)^{-1} S_{V G}^{-1}\right)^{3}
$$

Since $Q=q \cdot \gamma$, one can calculate the intergrain autocatalysis factor 


$$
\gamma=\left(\frac{\phi \cdot\left(1-V_{V G}\right) \ln \left(1-V_{V G}\right)^{-1}}{S_{V G} \cdot q^{1 / 3}}\right)^{3}
$$

To delve into the intragrain autocatalysis, consider that the spread events result from the intergrain transformation, and since it has been early accepted that martensite in steel is nucleation-controlled ${ }^{1,7,8}$, it is reasonable to relate $V_{V G}$ to the number density of martensite units in the material, $N_{V M}$, what amounts to relate the intragrain to the intergrain transformation. This has been accomplished by an equation proposed in ${ }^{16}$ based on computer simulation of the spreadevents and validated by describing data from "athermal" and "isothermal" martensitic transformations ${ }^{16,17,23}$

$$
V_{V G}=1-\exp \left(-\frac{q N_{V M}}{\beta}\right)
$$

where $\beta$ expresses the average the number of martensite units/grain necessary to spread the transformation into next grains. Phenomenologically, the Eq. (7) can be reconciled with Eq. (2) by equating $N_{V M}=\alpha . n_{V}$ where $\alpha$ is the overall autocatalytic factor (encompassing intergrain and intragrain processes). Substituting this relationship into Eq. (7) and comparing with Eq.(2) yields

$\alpha=\beta . \gamma$. Finally, considering that $\beta$ and $\gamma$ can be calculated with Eqs.(6-7) and $N_{V M}$ obtained directly from the microstructure, the number density of the initial nucleation sites can be determined

$$
n_{V}=N_{V M} \cdot(\beta \cdot \gamma)^{-1}
$$

\section{Results}

The values of $V_{V M}, N_{V M}, V_{V G}$ and $S_{V G}$ shown in Tables 1-2 were originally obtained by optical metallography 9 The previous equations were used to calculate the values of $\beta=\ln \left(1-V_{V G}\right)^{-1} / q N_{V M}, \gamma$ and of $n_{V}$, respectively. Assuming tetrakaidecahedron clusters and grains, we set $\varphi=16 / 3$ in Eq.(7), and the mean austenite grain volume $q=(4 . d / 3)^{3}$, where $d$ is the mean intercept length of the austenite grains. The values of $d$ are shown in the tables' captions. The temperatures in Tables 1-2 refer to the temperature of the quenching medium, $T$. Observe that values of $\gamma \gg 1$ are coherent with the development of spread-events.

\section{Discussion}

Tables 1-2 show that the number density of the martensite units, $N_{V M}$, are orders of magnitude higher than the calculated number density of initial martensite nucleation events, $n_{v}$, as might be expected. Moreover, the temperature dependence of $n_{V}$ fits the formalism advanced $\mathrm{in}^{24}$ to describe the results from Cech and Turnbull ${ }^{3}$ regarding martensite transformation in small particles - see Fig. 2 .

The slope of the fitted lines vary with the austenite grain size, reflecting a favorable environment for martensite nucleation ${ }^{25}$. Increasing the area of grain boundaries increases the possibility of initial nucleation events.

Inspection of Tables 1-2 shows that $\beta$ (thence intragrain autocatalysis) is not significantly affected by the transformation temperature. On the other hand, the intergrain process characterized by $Q$ exhibits Arrhenius behavior- see Fig.3. Despite the low fitting-correlation, attributable to the hardship associated with the characterization of the spread-events in the finer grained material, the small apparent activation energy, $E_{\mathrm{A}} \approx 2.810^{-20} \mathrm{~J} /$ event $\left(\mathrm{R}^{2}=0.55\right)$ compares with values typical of the dislocation mobility as well as with the activation energy for the thermal activated (isothermal) martensite nucleation ${ }^{26}$. Thermally activated stages in the transformation curve of a similar $\mathrm{Fe}-\mathrm{Ni}-\mathrm{C}$ alloy transformed by continuous cooling have already been identified and rationalized by admitting distinct potency distributions for the pre-existent and the autocatalytic nucleation $\operatorname{sites}^{27}$. In Figure 3, the Q values for both grain sizes were fitted together. The fitting of both sets of Q values together illustrates that the austenite grain size/mean intercept is not a relevant variable because the martensite units run across the grain boundaries. Villa et al. ${ }^{28}$ observed isothermal martensite kinetics on the isochronal cooling a Fe-Cr-Ni-Cu alloy which was attributed to thermally activated growth of athermal nucleated lath martensite. Laughlin et al. ${ }^{29}$ generalized the discussion of thermally-activated stages in the martensite transformation curve by considering the relative position of the $M_{S}$ temperature and the nose of the thermal-activated martensite transformation, and the imposed cooling rate. Here we provide evidence that the intergrain autocatalysis in polycrystalline $\mathrm{Fe}-31 \mathrm{wt} \% \mathrm{Ni}-0.02 \mathrm{wt} \% \mathrm{C}$ is controlled by a thermally activated process. The variations of $Q$ and $\gamma$

Table 1. Descriptors of the martensitic spread in Fe-31wt $\% \mathrm{Ni}-0.02 \mathrm{wt} \% \mathrm{C}$, mean intercept length equal to $0.048 \mathrm{~mm}$, transformed by quenching

\begin{tabular}{cccccccccc}
\hline$T(\mathrm{~K})$ & $V_{V M}$ & $N_{V M}\left(\mathrm{~mm}^{-3}\right)$ & $S_{V G}\left(\mathrm{~mm}^{-1}\right)$ & $V_{V G}$ & $n_{V}\left(\mathrm{~mm}^{-3}\right)$ & $\beta$ & $\gamma$ & $Q\left(\mathrm{~mm}^{3}\right)$ \\
\hline 220 & 0.05 & $9.10 \mathrm{E}+03$ & 2.9 & 0.23 & 5.12 & 9.13 & 195 & 0.051 \\
215 & 0.31 & $4.20 \mathrm{E}+04$ & 5.7 & 0.69 & 30.03 & 9.4 & 149 & 0.039 \\
213 & 0.4 & $4.60 \mathrm{E}+04$ & 5.7 & 0.73 & 36.37 & 9.21 & 137 & 0.036 \\
205 & 0.42 & $4.90 \mathrm{E}+04$ & 6.9 & 0.78 & 89.07 & 8.48 & 65 & 0.017 \\
203 & 0.37 & $7.80 \mathrm{E}+04$ & 6.9 & 0.75 & 72.96 & 14.75 & 72 & 0.019 \\
195 & 0.46 & $7.70 \mathrm{E}+04$ & 5.7 & 0.89 & 183.94 & 9.14 & 46 & 0.012 \\
188 & 0.47 & $1.30 \mathrm{E}+05$ & 4.9 & 0.9 & 143.91 & 14.8 & 61 & 0.016 \\
\hline
\end{tabular}


Table 2. Descriptors of the martensitic spread in $31 \mathrm{wt} \% \mathrm{Ni}-0.02 \mathrm{wt} \% \mathrm{C}$, mean intercept length equal to $0.027 \mathrm{~mm}$, transformed by quenching

\begin{tabular}{ccccccccc}
\hline$T(\mathrm{~K})$ & $V_{V M}$ & $N_{V M}\left(\mathrm{~mm}^{-3}\right)$ & $S_{V G}\left(\mathrm{~mm}^{-1}\right)$ & $V_{V G}$ & $n_{V}\left(\mathrm{~mm}^{-3}\right)$ & $\beta$ & $\gamma$ & $Q\left(\mathrm{~mm}^{3}\right)$ \\
\hline 212 & 0.04 & $2.71 \mathrm{E}+04$ & 1.4 & 0.11 & 2.01 & 10.85 & 1241 & 0.062 \\
208 & 0.16 & $9.50 \mathrm{E}+04$ & 8.4 & 0.33 & 76.62 & 11.07 & 112 & 0.005 \\
203 & 0.18 & $1.40 \mathrm{E}+05$ & 8.4 & 0.44 & 62.42 & 11.27 & 199 & 0.009 \\
196 & 0.26 & $1.60 \mathrm{E}+05$ & 10.7 & 0.49 & 126.56 & 11.09 & 114 & 0.005 \\
188 & 0.26 & $2.20 \mathrm{E}+05$ & 9.2 & 0.61 & 92.16 & 10.9 & 219 & 0.01 \\
183 & 0.39 & $3.00 \mathrm{E}+05$ & 12.2 & 0.72 & 317.12 & 11 & 86 & 0.004 \\
173 & 0.46 & $4.70 \mathrm{E}+05$ & 9.2 & 0.86 & 458.18 & 11.15 & 92 & 0.004 \\
\hline
\end{tabular}

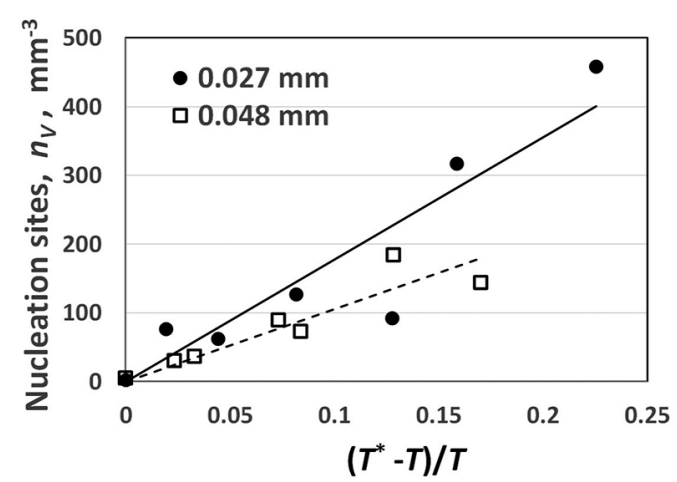

Figure 2. Fe-31wt $\% \mathrm{Ni}-0.02 \mathrm{wt} \% \mathrm{C}$ - Initial martensite nucleation sites calculated after Eq. (3), plotted after Guimarães and $\operatorname{Rios}^{24} ; T^{*} \cong M_{\mathrm{S}}$.

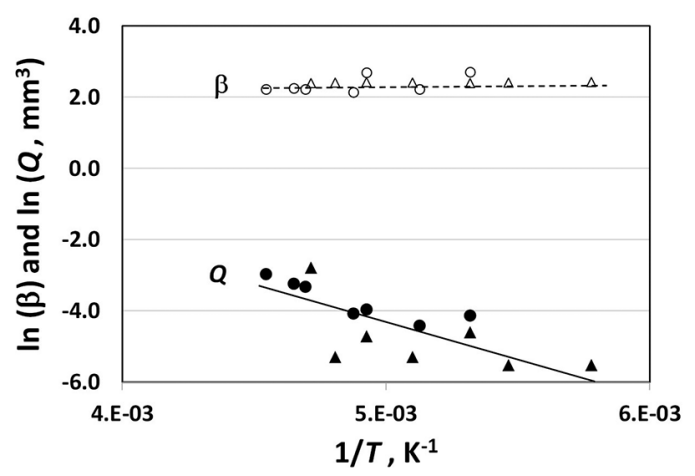

Figure 3. $\mathrm{Fe}-31 \mathrm{wt} \% \mathrm{Ni}-0.02 \mathrm{wt} \% \mathrm{C}$ - Evidence of thermally activated process step in the intergrain spread. Arrhenius plots of the $Q$ values, mean intercept length: $\bullet-0.048 \mathrm{~mm}$ and $\boldsymbol{\Delta}-0.027$ $\mathrm{mm}$, from Tables 1 and 2. Apparent activation energies $2.8 \times 10^{-20}$ $\mathrm{J} /$ event. The values of $\beta$, mean intercept length: $\odot-0.048 \mathrm{~mm}$ and $\Delta-0.027 \mathrm{~mm}$, are also shown.

in Tables 1-2 indicate the earlier exhaustion of the spreadevents at the lower temperatures, whereas the intragrain autocatalysis factor $\beta$ is nearly invariant.

To delve into the behavior of $\beta$ it is necessary to consider the interaction of martensite growth with the surrounding austenite. Haezebrouk ${ }^{30}$ simulations of martensite growth indicate that the extent of radial growth relates to the onset of a plastic zone ahead of the martensite rim. The latter depends on the stress threshold for austenite flow as well as on the martensite radial growth rate. It follows that intergrainautocatalysis is hindered if that plastic zone develops faster than a grain boundary impingement by a martensite unit can be effected. In the case of the Fe-31wt $\% \mathrm{Ni}-0.02 \mathrm{wt} \% \mathrm{C}$, it is apparent that impingements occur faster than the plastic zone develops in the adjacent austenite. This may be ascribed to the influence of the sub-zero temperature on the austenite plasticity. In this context, the auto-accommodation of the shape strain becomes crucial and can be accomplished by a local transformation burst comprising a limited number of $\beta$ units $^{12}$.

At this point it is relevant revisiting the influence of the austenite grain size on the extent of the intergrain autocatalysis in the $\mathrm{Fe}-23.2 \mathrm{wt} \% \mathrm{Ni}-2.8 \mathrm{wt} \% \mathrm{Mn}$ isothermal transformation described $\mathrm{in}^{31}$. In a previous review of the description of the isothermal with Eq. (7) we found out that the parameter $\beta$ decreases with increasing the ratio $D_{l} / d$. This highlights the drag effect of the $\mathrm{Fe}-23.2 \mathrm{wt} \% \mathrm{Ni}-2.8 \mathrm{wt} \% \mathrm{Mn}$ austenite on the radial growth of the martensite. The results are summarized in Table 3. Noteworthy, bearing the data in Figs. 9-10 $\mathrm{in}^{31}$, the initial scaled-diameter, $D_{l} / d$, of the Fe-23.2wt $\% \mathrm{Ni}-2.8 \mathrm{wt} \% \mathrm{Mn}$ martensite units increases on lowering the transformation temperature, whereas the scaled-thickness, $t_{l} / d$, decreases. This fact means that increasing the austenite strength delays the formation of the plastic zone that limits the radial growth of the martensite unit as discussed $\mathrm{in}^{30}$. The concomitant change in the composition of the units' invariant shear from slip-> twinning ${ }^{31,32}$ goes along with the change in the accommodation of the shape-strain.

Table 3. Fe-23.2wt $\% \mathrm{Ni}-2.8 \mathrm{wt} \% \mathrm{Mn}$ transformed at $153 \mathrm{~K}$.

\begin{tabular}{ccc}
\hline$D(\mathrm{~mm})^{*}$ & $\beta^{*}$ & $D / d$ \\
\hline 0.019 & 32.5 & 0.87 \\
0.048 & 96.1 & 0.56 \\
0.090 & 182 & 0.36 \\
\hline
\end{tabular}

* Same as in ref. ${ }^{30}$.

Last, the martensite-grain boundary impingement must be considered. The impact of a martensite units on a grain boundary produces a stress concentration within the region 
adjacent to the grain boundary. This stress can be relaxed by slip, by disruption of the grain boundary, or by stressassisted martensite transformation, which can occur on either side of the boundary ${ }^{25}$. Transformation induced within the neighbor grain by the plate impinging on the grain boundary is denominated "intergrain autocatalysis". The temperature dependence in $Q$ or $\gamma$, Fig. 3, highlights the importance of dislocation processes in such an event.

From the above one can infer that martensite autocatalysis results from the relaxation of transformation strains. Sympathetic nucleation and slip accomplish that. The former mechanically relaxes the shear-component of the shape-strain, promoting variant-selection. Complementary relaxation by slip introduces a thermally-activated dependence in the autocatalysis. Slip may become predominant when the austenite plasticity so permits, e.g. in materials that transform above the room temperature. It is worthy of note that in polycrystalline materials the austenite grain boundaries may become a hindrance to the transformation uniformity by limiting the size of the martensite units, as well as foster the intergrain-autocatalysis that spreads the transformation over the austenite grains.

\section{Summary and Conclusions}

This work provides a new appraisal of the martensite autocatalysis in $\mathrm{Fe}-31 \mathrm{wt} \% \mathrm{Ni}-0.02 \mathrm{wt} \% \mathrm{C}$ which has been one of the choice materials to investigate fundamental aspects of martensite transformations in iron base alloys. To cope with the typically fast transformation rate, we used a formalism that bears experimentally perceived aspects of the autocatalysis during the martensite spread. At martensite transformation temperatures a large driving force for austenite to martensite is available. However, owing to the peculiarities of martensite nucleation only a relatively small number of nuclei per unit of volume are normally available for nucleation of the socalled primary plates. To promote further transformation and therefore to achieve a larger decrease in Gibbs free energy, the martensite transformation "finds a way" resorting to autocatalysis to increase the number of potential nucleation sites from which new martensite plates form. Some specific conclusions are:

- In polycrystalline materials such as the $\mathrm{Fe}-31 \mathrm{wt} \% \mathrm{Ni}-$ $0.02 \mathrm{wt} \% \mathrm{C}$ alloy, the autocatalysis may combine intragrain and intergrain aspects, here described by the parameters, $\beta$ and $\gamma$ respectively. The intragrain transformation relates to the auto-accommodation of transformation strains, thence it has a mechanical aspect. The stress-assisted intergrain autocatalysis depends on thermal activation.

- $\quad$ The current analysis demonstrated that intragrain $(\beta)$ and intergrain $(\gamma)$ autocatalysis have a very different temperature dependence. On one hand, $\beta$ is independent of temperature and is possibly a function of mechanical autocatalysis (mutual accommodation of the shape strain) within the given austenite grain. On the other hand, $\gamma$ has an Arrhenius type temperature dependent with an activation energy compatible with dislocation processes within the austenite. This indicates that the intergrain autocatalysis is hindered at low temperatures.

- Thence, the autocatalysis provides further environments for martensite nucleation than are distinct than provided by the pre-existent austenitic sites. In a classical view, that is equivalent to admitting the autocatalytic nucleation sites with a distinct potency-distribution.

- The austenite grain boundaries have multiple effects in the martensite transformation, by fostering martensite nucleation, by limiting the size of the martensite units and by contributing to the intergrain autocatalysis (spread-events).

\section{Acknowledgements}

P. R. Rios is grateful to Conselho Nacional de Desenvolvimento Científico e Tecnológico, CNPq, and to Fundação de Amparo à Pesquisa do Estado do Rio de Janeiro, FAPERJ, for financial support. The authors are grateful to Prof. Kahl Zilnyk for providing the micrograph used in Fig. 1b.

\section{References}

1. Christian JW. Theory of Transformations in Metals and Alloys. $2^{\text {nd }}$ ed. Oxford: Pergamon Press; 1975.

2. Stojko A, Hansen MF, Slycke J, Somers MAJ. Isothermal martensite formation at sub-zero temperatures. Journal of ASTM International. 2011;8(4):1-9.

3. Cech RE, Turnbull D. Heterogeneous Nucleation of the Martensite Transformation. JOM. 1956;8(2):124-132.

4. Kastner O, Shneck RZ. On the entropic nucleation barrier in a martensitic transformation. Philosophical Magazine. 2015;95(12):1282-1308.

5. Zhang W, Jin YM, Khachaturyan AG. Phase field microelasticity modeling of heterogeneous nucleation and growth in martensitic alloys. Acta Materialia. 2007;55(2):565-574.

6. Shankaraiah N, Murthy KPN, Lookman T, Shenov SR. Incubation times and entropy barriers in martensitic kinetics: Monte Carlo quench simulation of strain pseudospins. EPL. 2010;92(3):36002.

7. Machlin ES, Cohen M. Burst phenomenon in the martensitic transformation. Transactions AIME. 1951;191(9):746-754.

8. Bunshah RF, Mehl RF. Rate of propagation of martensite. Transactions AIME. 1953;197:1251-1258.

9. Guimarães JRC, Gomes JC. Metallographic study of influence of austenite grain-size on martensite kinetics. Acta Metallurgica. 1978;26(10):1591-1596. 
10. Raghavan V. Formation sequence of plates in isothermal martensite transformation. Acta Metallurgica. 1969;17(10):1299-1303.

11. Guimarães JRC, Rios PR. Microstructural Analysis of Martensite Dimensions in FeNiC and $\mathrm{FeC}$ Alloys. Materials Research. 2015;18(3)595-601.

12. Bokros JC, Parker ER. The mechanism of the martensite burst transformation in Fe-Ni single crystals. Acta Metallurgica. 1963;11(12):1291-1301.

13. Kundu S, Verma AK, Sharma V. Quantitative Analysis of Variant Selection for Displacive Transformations Under Stress. Metallurgical and Materials Transactions A . 2012;43(7):25522565 .

14. Steele JH, McCall JL, eds. Practical Applications of Quantitative Metallography. STP 839. Philadelphia: ASTM; 1984.

15. Fullman RL. Measurement of particle sizes in opaque bodies. Transactions AIME. 1953;197(3):447-452.

16. Guimarães JRC, Saavedra A. A computer-assisted analysis of the spread of martensite-transformation. Materials Science and Engineering. 1984;62(1):11-15.

17. Rios PR, Guimarães JRC. Microstructural path analysis of athermal martensite. Scripta Materialia. 2007;57(12):11051108 .

18. Johnson WA, Mehl RF. Reaction kinetics in processes of nucleation and growth. Transactions AIME. 1939;135:416-441.

19. Avrami MJ. Kinetics of Phase Change I General Theory. The Journal of Chemical Physics. 1939;7(12):1103-1112.

20. Kolmogorov AN. The statistics of crystal growth in metals. Isvestiia Academii Nauk SSSR - Seriia Matematicheskaia. 1937;1:333-359.

21. Vandermeer RA. Microstructural descriptors and the effects of nuclei clustering on recrystallization path kinetics. Acta Materialia. 2005;53(5):1449-1457.
22. Rios PR, Guimarães JRC. Formal analysis of isothermal martensite spread. Materials Research. 2008;11(1):103-108.

23. Guimarães JRC. Isothermal martensite: the austenite grain-size and the kinetics of "spread". Materials Science and Technology. 2008;24(7):843-847.

24. Guimarães JRC, Rios PR. Initial nucleation kinetics of martensite transformation. Journal of Materials Science. 2008;43(15):52065210 .

25. Heo TW, Chen LQ. Phase-field modeling of displacive phase transformations in elastically anisotropic and inhomogeneous polycrystals. Acta Materialia. 2014;76:68-81.

26. Pati SR, Cohen M. Nucleation of isothermal martensitic transformation. Acta Metallurgica. 1969;17(3):189-199.

27. Lin M, Olson GB, Cohen M. Distributed-activation kinetics of heterogeneous martensitic nucleation. Metallurgical and Materials Transactions A. 1992;23(11):2987-2998.

28. Villa M, Pantleon K, Reich M, Kessler O, Somers MAJ. Kinetics of anomalous multi-step formation of lath martensite in steel. Acta Materialia. 2014;80(11):468-477.

29. Laughlin DE, Jones NJ, Schwartz AJ, Massalski TB. Thermally activated martensite: its relationship to non-thermally activated (athermal) martensite. In: Olson GB, Lieberman DS, Saxena A, eds. International Conference on Martensitic Transformations (ICOMAT). Hoboken: Wiley; 2010. p. 141-143.

30. Haezebrouck DM. Nucleation and growth of a single martensite particle. [Thesis]. Cambridge: Massachusetts Institute of Technology; 1987.

31. Ghosh G. Spread of transformation and plate dimensions of isothermally formed martensite. Materials Science and Engineering: A. 1988;101:213-220.

32. Ghosh G, Raghavan V. The dimensions of isothermally formed martensitic plates in a Fe-Ni-Mn alloy. Materials Science and Engineering. 1986;79(2)223-231. 